\title{
Erratum to: Phospholipase $C-\eta 2$ interacts with nuclear and cytoplasmic LIMK-1 during retinoic acid-stimulated neurite growth
}

\author{
Mohammed Arastoo $^{1}$ - Christian Hacker ${ }^{1,2} \cdot$ Petra Popovics $^{1,3} \cdot$ John M. Lucocq $^{1}$. \\ Alan J. Stewart ${ }^{1}$
}

Published online: 12 February 2016

(C) Springer-Verlag Berlin Heidelberg 2016

\section{Erratum to: Histochem Cell Biol \\ (2016) 145:163-173 \\ DOI 10.1007/s00418-015-1390-7}

Unfortunately, in the original publication of the article, the sentences "We first investigated the role of the PLC $\eta 2$ calcium-binding EF-hand domain, a domain that is known to be required for PLC $\eta 2$ activation" and "Taken together, these data emphasize the importance of the PLC $\eta 2$ EFhand domain and articulation of PLC $\eta 2$ with LIMK-1 in regulating neuritogenesis" were incorrectly published in the abstract section.

The correct sentences should read as follows:

"We first investigated the role of the PLC $\eta 2$ calcium-binding EF-hand domain, a domain that is known to be required for calcium-dependent PLC $\eta 2$ activation" and

"Taken together, these data emphasize the importance of PLC $\eta 2$ and its articulation with LIMK-1 in regulating neuritogenesis."

The online version of the original article can be found under doi:10.1007/s00418-015-1390-7.

Alan J. Stewart

ajs21@st-andrews.ac.uk

1 School of Medicine, Medical and Biological Sciences Building, North Haugh, University of St Andrews, St Andrews, Fife KY16 9TF, UK

2 Bioimaging Centre, Geoffrey Pope Building, College of Life and Environmental Sciences, University of Exeter, Exeter EX4 4QD, UK

3 Veterans Affairs Medical Center, Miami, FL 33125, USA 